\title{
On a System of Volterra Type Hadamard Fractional Integral Equations in Fréchet Spaces
}

\author{
Saïd Abbas $\mathbb{D}^{1},{ }^{1}$ Mouffak Benchohra $\mathbb{D}^{\mathbb{D}},{ }^{2}$ and Yong Zhou $\mathbb{D}^{3,4}$ \\ ${ }^{1}$ Laboratory of Mathematics, Tahar Moulay University of Saïda, Saïda 20000, Algeria \\ ${ }^{2}$ Laboratory of Mathematics, Djillali Liabes University of Sidi Bel-Abbès, Sidi Bel-Abbès 22000, Algeria \\ ${ }^{3}$ Faculty of Information Technology, Macau University of Science and Technology, Macau 999078, China \\ ${ }^{4}$ Faculty of Mathematics and Computational Science, Xiangtan University, Hunan 411105, China
}

Correspondence should be addressed to Yong Zhou; yzhou@xtu.edu.cn

Received 9 May 2018; Accepted 30 October 2018; Published 11 November 2018

Academic Editor: Josef Diblík

Copyright (C) 2018 Saïd Abbas et al. This is an open access article distributed under the Creative Commons Attribution License, which permits unrestricted use, distribution, and reproduction in any medium, provided the original work is properly cited.

In this article we present some results concerning the existence of solutions for a system of Hadamard integral equations. Our investigation is conducted with an application of an extension of the fixed point theorem of Burton-Kirk in Fréchet spaces.

\section{Introduction}

In recent years, fractional differential and integral equations are emerging as a useful tool in modeling of the dynamics of many physical systems and electrical phenomena, which has been demonstrated by many researchers in the fields of mathematics, science, and engineering; see monographs [1-7] and a series of papers [8-18] and the references cited therein. Some properties of the Hadamard fractional calculus were investigated by Butzer et al. [19,20]. Additional papers can be found in $[7,21]$ and the references therein. Recently, some interesting results on the existence of the solutions of some classes of integral equations have been obtained by Abbas et al. [22-24], Banaś et al. [25, 26], and Pachpatte $[27,28]$ and the references therein.

This paper deals with the existence of solutions of the following system of Hadamard fractional integral equations of the form

$$
\begin{gathered}
u_{1}(t, x)=\mu_{1}(t, x)+f_{1}\left(t, x,\left({ }^{H} I_{\sigma}^{r} u_{1}\right)(t, x), u_{1}(t, x)\right) \\
+\frac{1}{\Gamma\left(r_{1}\right) \Gamma\left(r_{2}\right)} \int_{1}^{t} \int_{1}^{x}\left(\log \frac{t}{s}\right)^{r_{1}-1}\left(\log \frac{x}{y}\right)^{r_{2}-1} \times g_{1}(t, \\
\left.x, s, y, u_{1}(s, y), u_{2}(s, y), \ldots, u_{n}(s, y)\right) \frac{d y d s}{s y} \\
u_{2}(t, x)=\mu_{1}(t, x)+f_{2}\left(t, x,\left({ }^{H} I_{\sigma}^{r} u_{2}\right)(t, x), u_{2}(t, x)\right)
\end{gathered}
$$

$$
\begin{aligned}
& +\frac{1}{\Gamma\left(r_{1}\right) \Gamma\left(r_{2}\right)} \int_{1}^{t} \int_{1}^{x}\left(\log \frac{t}{s}\right)^{r_{1}-1}\left(\log \frac{x}{y}\right)^{r_{2}-1} \times g_{2}(t, \\
& \left.x, s, y, u_{1}(s, y), u_{2}(s, y), \ldots, u_{n}(s, y)\right) \frac{d y d s}{s y} \\
& \ldots \ldots \ldots \ldots \\
& u_{n}(t, x)=\mu_{n}(t, x)+f_{n}\left(t, x,\left({ }^{H} I_{\sigma}^{r} u_{n}\right)(t, x), u_{n}(t, x)\right) \\
& +\frac{1}{\Gamma\left(r_{1}\right) \Gamma\left(r_{2}\right)} \int_{1}^{t} \int_{1}^{x}\left(\log \frac{t}{s}\right)^{r_{1}-1}\left(\log \frac{x}{y}\right)^{r_{2}-1} \\
& \times g_{n}\left(t, x, s, y, u_{1}(s, y), u_{2}(s, y), \ldots,\right. \\
& \left.u_{n}(s, y)\right) \frac{d y d s}{s y} ; \quad(t, x) \in J:=[1,+\infty) \times[1, b] .
\end{aligned}
$$

That is,

$$
\begin{gathered}
u_{i}(t, x)=\mu_{i}(t, x)+f_{i}\left(t, x,\left({ }^{H} I_{\sigma}^{r} u_{i}\right)(t, x), u_{i}(t, x)\right) \\
+\frac{1}{\Gamma\left(r_{1}\right) \Gamma\left(r_{2}\right)} \int_{1}^{t} \int_{1}^{x}\left(\log \frac{t}{s}\right)^{r_{1}-1}\left(\log \frac{x}{y}\right)^{r_{2}-1} \times g_{i}(t,
\end{gathered}
$$




$$
\begin{array}{r}
\left.x, s, y, u_{1}(s, y), u_{2}(s, y), \ldots, u_{n}(s, y)\right) \frac{d y d s}{s y} ; \\
(t, x) \in J, i=1, \ldots, n,
\end{array}
$$

where $b>1, \sigma=(1,1), r=\left(r_{1}, r_{2}\right), r_{1}, r_{2} \in(0, \infty),{ }^{H} I_{\sigma}^{r}$ is the Hadamard integral of order $r, \mu_{i}: J \longrightarrow \mathbb{R}, f_{i}: J \times$ $\mathbb{R}^{2} \longrightarrow \mathbb{R}, g_{i}: J^{\prime} \times \mathbb{R}^{n} \longrightarrow \mathbb{R}$ are given continuous functions, $J^{\prime}=\left\{(t, x, s, y) \in J^{2}: s \leq t, y \leq x\right\}$, and $\Gamma(\cdot)$ is the (Euler's) gamma function defined by

$$
\Gamma(\xi)=\int_{0}^{\infty} t^{\xi-1} e^{-t} d t ; \quad \xi>0 .
$$

\section{Preliminaries}

Let $L^{1}([1,+\infty) \times[1, b])$; for $b>1$, be the space of Lebesgueintegrable functions $u:[1,+\infty) \times[1, b] \longrightarrow \mathbb{R}$ with the standard norm

$$
\|u\|_{1}=\int_{1}^{\infty} \int_{1}^{b}|u(t, x)| d x d t .
$$

Definition 1 (see [4]). The Hadamard fractional integral of order $q>0$ for a function $g \in L^{1}([1, a], \mathbb{R})$ is defined as

$$
\left({ }^{H} I_{1}^{q} g\right)(x)=\frac{1}{\Gamma(q)} \int_{1}^{x}\left(\log \frac{x}{s}\right)^{q-1} \frac{g(s)}{s} d s .
$$

Example 2. The Hadamard fractional integral of order $q>0$ for the function $w:[1, e] \longrightarrow \mathbb{R}$, defined by $w(x)=(\log x)^{\beta-1}$ with $\beta>0$, is

$$
\left({ }^{H} I_{1}^{q} w\right)(x)=\frac{\Gamma(\beta)}{\Gamma(\beta+q)}(\log x)^{\beta+q-1} .
$$

Definition 3. Let $r_{1}, r_{2} \geq 0, \sigma=(1,1)$, and $r=\left(r_{1}, r_{2}\right)$. For $w \in L^{1}(J, \mathbb{R})$, define the Hadamard partial fractional integral of order $r$ by the expression

$$
\begin{aligned}
& \left({ }^{H} I_{\sigma}^{r} w\right)(x, y)=\frac{1}{\Gamma\left(r_{1}\right) \Gamma\left(r_{2}\right)} \\
& \quad \cdot \int_{1}^{x} \int_{1}^{y}\left(\log \frac{x}{s}\right)^{r_{1}-1}\left(\log \frac{y}{t}\right)^{r_{2}-1} \frac{w(s, t)}{s t} d t d s .
\end{aligned}
$$

Let $X$ be a Fréchet space with a family of seminorms $\left\{\|\cdot\|_{n}\right\}_{n \in \mathbb{N}^{*}}$ such that

$$
\|x\|_{1} \leq\|x\|_{2} \leq\|x\|_{3} \leq \ldots \quad \text { for every } x \in X .
$$

A subset $Y$ of $X$ is bounded if, for every $n \in \mathbb{N}$, there exists $\bar{M}_{n}>0$ such that

$$
\|y\|_{n} \leq \bar{M}_{n} \quad \text { for all } y \in Y .
$$

We associate a sequence of Banach spaces $\left\{\left(X^{n},\|\cdot\|_{n}\right)\right\}$ to $X$ as follows: For every $n \in \mathbb{N}$, we consider the equivalence relation $\sim_{n}$ defined by $x \sim_{n} y$ if and only if $\|x-y\|_{n}=0$ for $x, y \in X$.
We denote by $X^{n}=\left(\left.X\right|_{\sim_{n}},\|\cdot\|_{n}\right)$ the quotient space, the completion of $X^{n}$ with respect to $\|\cdot\|_{n}$. To every $Y \subset X$, we associate a sequence $\left\{Y^{n}\right\}$ of subsets $Y^{n} \subset X^{n}$ as follows: For every $x \in X$, we denote by $[x]_{n}$ the equivalence class of $x$ of subset $X^{n}$ and we defined $Y^{n}=\left\{[x]_{n}: x \in Y\right\}$. We denote by $\overline{Y^{n}}$, int $_{n}\left(Y^{n}\right)$, and $\partial_{n} Y^{n}$, respectively, the closure, the interior, and the boundary of $Y^{n}$ with respect to $\|\cdot\|_{n}$ in $X^{n}$. For more details, we refer the reader to [29].

Definition 4. Let $X$ be a Fréchet space. A function $N: X \longrightarrow$ $X$ is said to be a contraction if for each $n \in \mathbb{N} \backslash\{0\}$ there exists $k_{n} \in[0,1)$ such that

$$
\|N(u)-N(v)\|_{n} \leq k_{n}\|u-v\|_{n} \quad \text { for all } u, v \in X .
$$

We need the following extension of the Burton-Kirk fixed point theorem in the case of a Fréchet space.

Theorem 5 (see [30]). Let $\left(X,\|\cdot\|_{n}\right)$ be a Fréchet space and let $A, B: X \longrightarrow X$ be two operators such that

(a) $A$ is a compact operator

(b) $B$ is a contraction operator with respect to a family of seminorms $\left\{\|\cdot\|_{n}\right\}$

(c) the set $\{x \in X: x=\lambda A(x)+\lambda B(x / \lambda), \lambda \in(0,1)\}$ is bounded

Then the operator equation $A(u)+B(u)=u$ has a solution in $X$.

\section{Existence Results}

Set

$$
C^{n}(J)=C(J) \times \ldots \times C(J) \quad \text { (n times) } .
$$

Definition 6. A function $\left(u_{1}, u_{2}, \ldots, u_{n}\right) \in C^{n}(J)$ is said to be a solution of (2) if $\left(u_{1}, u_{2}, \ldots, u_{n}\right)$ satisfies (2) on $J$.

For each $p \in \mathbb{N} \backslash\{0,1\}$ we consider the set $C_{p}=C([1, p] \times$ $[1, b])$, and we define in $C(J)$ the seminorms by

$$
\|u\|_{p}=\sup _{(t, x) \in[1, p] \times[1, b]}|u(t, x)| .
$$

Then $C$ is a Fréchet space with the family of seminorms $\left\{\|u\|_{p}\right\}$.

Also, the product space $\mathscr{C}:=C^{n}$ is a Fréchet space with the family of seminorms

$$
\left\|\left(u_{1}, u_{2}, \ldots, u_{n}\right)\right\|_{p}=\sum_{i=1}^{n}\left\|u_{i}\right\|_{p} .
$$

Now, we are concerned with the existence of solutions for system (2). Let us introduce the following hypotheses:

$\left(H_{1}\right)$ There exist continuous functions $l_{i}, k_{i}: J \longrightarrow \mathbb{R}_{+} ; i=$ $1, \ldots, n$, with

$$
\lim _{t \rightarrow \infty} l_{i}(t, x)=\lim _{t \longrightarrow \infty} k_{i}(t, x)=0 ; \quad \text { for } x \in[1, b] \text {, }
$$


such that

$$
\begin{aligned}
& \left|f_{i}\left(t, x, u_{1}, v_{1}\right)-f_{i}\left(t, x, u_{2}, v_{2}\right)\right| \\
& \quad \leq \frac{l_{i}(t, x)\left|u_{1}-u_{2}\right|+k_{i}(t, x)\left|v_{1}-v_{2}\right|}{1+\left|u_{1}-u_{2}\right|+\left|v_{1}-v_{2}\right|},
\end{aligned}
$$

for each $(t, x) \in J$ and each $u_{1}, u_{2}, v_{1}, v_{2} \in \mathbb{R}$

$\left(H_{2}\right)$ There exist continuous functions $Q_{i j}, \varphi_{i}: J^{\prime} \longrightarrow \mathbb{R}_{+}$; $i=1, \ldots, n, j=0, \ldots, n$, and nondecreasing functions $\psi_{i}:[0, \infty) \longrightarrow(0, \infty)$ such that

$$
\begin{aligned}
& \left|g_{i}\left(t, x, s, y, u_{1}, u_{2}, \ldots, u_{n}\right)\right| \\
& \quad \leq \frac{Q_{i 0}(t, x, s, y)+\sum_{j=1}^{n} Q_{i j}(t, x, s, y)\left|u_{j}\right|}{1+\sum_{j=1}^{n}\left|u_{j}\right|}
\end{aligned}
$$

for $(t, x, s, y) \in J^{\prime}, u_{i} \in \mathbb{R} ; i=1, \ldots, n$, and

$$
\begin{aligned}
& \left|g_{i}\left(t_{1}, x_{1}, s, y, u_{1}, \ldots, u_{n}\right)-g_{i}\left(t_{2}, x_{2}, s, y, u_{1}, \ldots, u_{n}\right)\right| \\
& \quad \leq \varphi_{i}(s, y)\left(\left|t_{1}-t_{2}\right|+\left|x_{1}-x_{2}\right|\right) \times \sum_{j=1}^{n} \psi_{i}\left(\left|u_{j}\right|\right) ;
\end{aligned}
$$$$
\left(t_{1}, x_{1}\right),\left(t_{2}, x_{2}\right),(s, y) \in J, u_{i} \in \mathbb{R} \text {. }
$$

Moreover, assume that

$$
\lim _{t \rightarrow \infty} \int_{1}^{t}\left|\log \frac{t}{s}\right|^{r_{1}-1} \frac{Q_{i j}(t, x, s, y)}{s} d s=0,
$$

for each $(x, y) \in J$ with $y \leq x$

For any $p \in \mathbb{N} \backslash\{0,1\}$, set

$$
k_{p}^{*}=\max _{i=1, \ldots, n} k_{i p}=\max _{i=1, \ldots, n} \sup _{(t, x) \in[1, p] \times[1, b]} k_{i}(t, x),
$$

and

$$
l_{p}^{*}=\max _{i=1, \ldots, n} l_{i p}=\max _{i=1, \ldots, n} \sup _{(t, x) \in[1, p] \times[1, b]} l_{i}(t, x) .
$$

Theorem 7. Assume that hypotheses $\left(H_{1}\right)$ and $\left(H_{2}\right)$ hold. If

$$
L_{p}:=k_{p}^{*}+\frac{l_{p}^{*}(\log p)^{r_{1}}(\log b)^{r_{2}}}{\Gamma\left(1+r_{1}\right) \Gamma\left(1+r_{2}\right)}<1,
$$

then system (2) has at least one solution in the space $\mathscr{C}$.

Proof. Define the operators $A_{i}, B_{i}: C \longrightarrow C ; i=1, \ldots, n$, by

$$
\begin{gathered}
\left(A_{i} u_{i}\right)(t, x)=\int_{1}^{t} \int_{1}^{x}\left(\log \frac{t}{s}\right)^{r_{1}-1}\left(\log \frac{x}{y}\right)^{r_{2}-1} \\
\times \frac{g_{i}\left(t, x, s, y, u_{1}(s, y), \ldots, u_{n}(s, y)\right)}{s y \Gamma\left(r_{1}\right) \Gamma\left(r_{2}\right)} d y d s ;
\end{gathered}
$$

$$
(t, x) \in J
$$

$$
\left(B_{i} u_{i}\right)(t, x)=\mu_{i}(t, x)+f_{i}\left(t, x,{ }^{H} I_{\sigma}^{r} u_{i}(t, x), u_{i}(t, x)\right) ;
$$

$$
(t, x) \in J
$$

and consider the continuous operators $A, B: \mathscr{C} \longrightarrow \mathscr{C}$ defined by

$$
\begin{aligned}
& A\left(u_{1}, \ldots, u_{n}\right)=\left(A_{1}\left(u_{1}\right), \ldots, A_{n}\left(u_{n}\right)\right), \\
& B\left(u_{1}, \ldots, u_{n}\right)=\left(B_{1}\left(u_{1}\right), \ldots, B_{n}\left(u_{n}\right)\right) .
\end{aligned}
$$

We shall show that operators $A$ and $B$ satisfied all the conditions of Theorem 5 .

The proof will be given in several steps.

Step 1 ( $A$ is compact). To this aim, we must prove that $A$ is continuous and it transforms every bounded set into a relatively compact set. Recall that $M \subset \mathscr{C}$ is bounded if and only if

$$
\begin{gathered}
\forall p \in \mathbb{N} \backslash\{0,1\}, \\
\exists \ell_{p}>0: \forall\left(u_{1}, \ldots, u_{n}\right) \in M, \\
\left\|\left(u_{1}, \ldots, u_{n}\right)\right\|_{p} \leq \ell_{p},
\end{gathered}
$$

and $\left.M=\left\{\left(u_{1}(t, x), \ldots, u_{n}(t, x)\right) ;(t, x)\right) \in J\right\} \subset \mathscr{C}$ is relatively compact if and only if, $\forall p \in \mathbb{N} \backslash\{0,1\}$, the family $\left\{\left.\left(u_{1}(t, x), \ldots, u_{n}(t, x)\right)\right|_{(t, x)] \in[1, p] \times[1, b]}\right\}$ is equicontinuous and uniformly bounded on $[1, p] \times[1, b]$. The proof will be given in several claims.

Claim 1 ( $A$ is continuous). Let $\left\{\left(u_{1 m}, \ldots, u_{n m}\right)\right\}_{m \in \mathbb{N} \backslash\{0,1\}}$ be a sequence in $M$ such that $\left(u_{1 m}, \ldots, u_{n m}\right) \longrightarrow\left(u_{1}, \ldots, u_{n}\right)$ in $M$. Then, for each $(t, x) \in J$ and $i=1, \ldots, n$, we have

$$
\begin{aligned}
& \left|\left(A_{i}\left(u_{i m}\right)\right)(t, x)-\left(A\left(u_{i}\right)\right)(t, x)\right| \leq \frac{1}{\Gamma\left(r_{1}\right) \Gamma\left(r_{2}\right)} \\
& \quad . \int_{1}^{t} \int_{1}^{x}\left|\log \frac{t}{s}\right|^{r_{1}-1}\left|\log \frac{x}{y}\right|^{r_{2}-1} \\
& \quad \times \mid g_{i}\left(t, x, s, y, u_{1 m}(s, y), \ldots, u_{n m}(s, y)\right) \\
& -g_{i}\left(t, x, s, y, u_{1}(s, y), \ldots, u_{n}(s, y)\right) \mid d y d s \\
& \quad \leq \frac{1}{\Gamma\left(r_{1}\right) \Gamma\left(r_{2}\right)} \int_{1}^{t} \int_{1}^{x}\left|\log \frac{t}{s}\right|^{r_{1}-1}\left|\log \frac{x}{y}\right|^{r_{2}-1} \\
& \quad \times \mid g_{i}\left(t, x, s, y, u_{1 m}(s, y), \ldots, u_{n m}(s, y)\right) \\
& \quad-g_{i}\left(t, x, s, y, u_{1}(s, y), \ldots, u_{n}(s, y)\right) \mid d y d s .
\end{aligned}
$$

Since $u_{i m} \longrightarrow u_{i}$ as $m \longrightarrow \infty$ and $g_{i}$ is continuous, then for each $(t, x) \in[1, p] \times[1, b] ; p \in \mathbb{N} \backslash\{0,1\}$, and $i=1, \ldots, n$, (27) gives

$$
\begin{aligned}
& \left\|A\left(u_{1 m}, \ldots, u_{n m}\right)-A\left(u_{1}, \ldots, u_{n}\right)\right\|_{p} \\
& \quad=\sum_{i=1}^{n}\left\|A_{i}\left(u_{i m}\right)-A_{i}\left(u_{i}\right)\right\|_{p} \longrightarrow 0 \text { as } m \longrightarrow \infty .
\end{aligned}
$$

Claim 2 ( $A$ maps bounded sets into bounded sets in $C$ ). Let $M$ be the bounded set in $\mathscr{C}$ as in Claim 1. Then, for each 
$p \in \mathbb{N} \backslash\{0,1\}$, there exists $\ell_{p}>0$, such that for all $\left(u_{1}, \ldots, u_{n}\right) \in \mathscr{C}$ we have $\left\|\left(u_{1}, \ldots, u_{n}\right)\right\|_{p} \leq \ell_{p}$.

For arbitrarily fixed $(t, x) \in[1, p] \times[1, b]$ and each $i=$ $1, \ldots, n$, we have

$$
\begin{aligned}
& \left|\left(A_{i} u_{i}\right)(t, x)\right| \leq \frac{1}{\Gamma\left(r_{1}\right) \Gamma\left(r_{2}\right)} \int_{1}^{t} \int_{1}^{x}\left|\log \frac{t}{s}\right|^{r_{1}-1}\left|\log \frac{x}{y}\right|^{r_{2}-1} \\
& \quad \times\left|g_{i}\left(t, x, s, y, u_{1}(s, y), \ldots, u_{n}(s, y)\right)\right| d y d s \\
& \quad \leq \frac{1}{\Gamma\left(r_{1}\right) \Gamma\left(r_{2}\right)} \int_{1}^{t} \int_{1}^{x}\left|\log \frac{t}{s}\right|^{r_{1}-1}\left|\log \frac{x}{y}\right|^{r_{2}-1} \\
& \quad \times \frac{Q_{i 0}(t, x, s, y)+\sum_{j=1}^{n} Q_{i j}(t, x, s, y)|u(s, y)|}{1+\sum_{j=1}^{n}\left|u_{j}(s, y)\right|} d y d s \\
& \quad \leq \frac{1}{\Gamma\left(r_{1}\right) \Gamma\left(r_{2}\right)} \int_{1}^{t} \int_{1}^{x}\left|\log \frac{t}{s}\right|^{r_{1}-1}\left|\log \frac{x}{y}\right|^{r_{2}-1} \\
& \quad \times\left(\sum_{j=0}^{n} Q_{i j}(t, x, s, y)\right) d y d s \leq Q_{i p},
\end{aligned}
$$

where

$$
\begin{aligned}
Q_{i p} & =\sup _{(t, x) \in[1, p] \times[1, b]} \int_{1}^{t} \int_{1}^{x}\left|\log \frac{t}{s}\right|^{r_{1}-1}\left|\log \frac{x}{y}\right|^{r_{2}-1} \\
& \frac{\sum_{j=0}^{n} Q_{i j}(t, x, s, y)}{\Gamma\left(r_{1}\right) \Gamma\left(r_{2}\right)} d y d s .
\end{aligned}
$$

Hence

$$
\left\|A\left(u_{1}, \ldots, u_{n}\right)\right\|_{p} \leq \sum_{i=0}^{n} Q_{i p}:=Q_{p}^{*} .
$$

Claim 3 ( $A$ maps bounded sets into equicontinuous sets in $C$ ). Let $\left(t_{1}, x_{1}\right),\left(t_{2}, x_{2}\right) \in[1, p] \times[1, b], t_{1}<t_{2}, x_{1}<x_{2}$, and let $u \in M$; thus for each $i=1, \ldots, n$, we have

$$
\begin{aligned}
& \left|\left(A_{i} u_{i}\right)\left(t_{2}, x_{2}\right)-\left(A_{i} u_{i}\right)\left(t_{1}, x_{1}\right)\right| \leq\left.\frac{1}{\Gamma\left(r_{1}\right) \Gamma\left(r_{2}\right)}\left|\int_{1}^{t_{2}} \int_{1}^{x_{2}}\right| \log \frac{t_{2}}{s}\right|^{r_{1}-1}\left|\log \frac{x_{2}}{y}\right|^{r_{2}-1} \\
& \times\left[g_{i}\left(t_{2}, x_{2}, s, y, u_{1}(s, y), \ldots, u_{n}(s, y)\right)-g_{i}\left(t_{1}, x_{1}, s, y, u_{1}(s, y), \ldots, u_{n}(s, y)\right)\right] d y d s \\
& +\left.\frac{1}{\Gamma\left(r_{1}\right) \Gamma\left(r_{2}\right)}\left|\int_{1}^{t_{2}} \int_{1}^{x_{2}}\right| \log \frac{t_{2}}{s}\right|^{r_{1}-1}\left|\log \frac{x_{2}}{y}\right|^{r_{2}-1} g_{i}\left(t_{1}, x_{1}, s, y, u_{1}(s, y), \ldots, u_{n}(s, y)\right) d y d s \\
& -\int_{1}^{t_{2}} \int_{1}^{x_{2}}\left|\log \frac{t_{1}}{s}\right|^{r_{1}-1}\left|\log \frac{x_{1}}{y}\right|^{r_{2}-1} g_{i}\left(t_{1}, x_{1}, s, y, u_{1}(s, y), \ldots, u_{n}(s, y)\right) d y d s \mid \\
& +\left.\frac{1}{\Gamma\left(r_{1}\right) \Gamma\left(r_{2}\right)}\left|\int_{1}^{t_{2}} \int_{1}^{x_{2}}\right| \log \frac{t_{1}}{s}\right|^{r_{1}-1}\left|\log \frac{x_{1}}{y}\right|^{r_{2}-1} g_{i}\left(t_{1}, x_{1}, s, y, u_{1}(s, y), \ldots, u_{n}(s, y)\right) d y d s \\
& -\left.\int_{1}^{t_{1}} \int_{1}^{x_{1}}\left|\log \frac{t_{1}}{s}\right|^{r_{1}-1}\left|\log \frac{x_{1}}{y}\right|^{r_{2}-1} g_{i}\left(t_{1}, x_{1}, s, y, u_{1}(s, y), \ldots, u_{n}(s, y)\right) d y d s\left|\leq \frac{1}{\Gamma\left(r_{1}\right) \Gamma\left(r_{2}\right)} \int_{1}^{t_{2}} \int_{1}^{x_{2}}\right| \log \frac{t_{2}}{s}\right|^{r_{1}-1} \\
& \cdot\left|\log \frac{x_{2}}{y}\right|^{r_{2}-1} \times\left|g_{i}\left(t_{2}, x_{2}, s, y, u_{1}(s, y), \ldots, u_{n}(s, y)\right)-g_{i}\left(t_{1}, x_{1}, s, y, u_{1}(s, y), \ldots, u_{n}(s, y)\right)\right| d y d s \\
& +\frac{1}{\Gamma\left(r_{1}\right) \Gamma\left(r_{2}\right)} \int_{1}^{t_{1}} \int_{1}^{x_{1}}\left|\left(\log \frac{t_{2}}{s}\right)^{r_{1}-1}\left(\log \frac{x_{2}}{y}\right)^{r_{2}-1}-\left(\log \frac{t_{1}}{s}\right)^{r_{1}-1}\left(\log \frac{x_{1}}{y}\right)^{r_{2}-1}\right| \\
& \cdot\left|g_{i}\left(t_{1}, x_{1}, s, y, u_{1}(s, y), \ldots, u_{n}(s, y)\right)\right| d y d s+\frac{1}{\Gamma\left(r_{1}\right) \Gamma\left(r_{2}\right)} \int_{1}^{t_{1}} \int_{x_{1}}^{x_{2}}\left|\log \frac{t_{2}}{s}\right|^{r_{1}-1}\left|\log \frac{x_{2}}{y}\right|^{r_{2}-1} \\
& \cdot\left|g_{i}\left(t_{1}, x_{1}, s, y, u_{1}(s, y), \ldots, u_{n}(s, y)\right)\right| d y d s+\frac{1}{\Gamma\left(r_{1}\right) \Gamma\left(r_{2}\right)} \int_{t_{1}}^{t_{2}} \int_{1}^{x_{1}}\left|\log \frac{t_{2}}{s}\right|^{r_{1}-1}\left|\log \frac{x_{2}}{y}\right|^{r_{2}-1} \\
& \cdot\left|g_{i}\left(t_{1}, x_{1}, s, y, u_{1}(s, y), \ldots, u_{n}(s, y)\right)\right| d y d s+\frac{1}{\Gamma\left(r_{1}\right) \Gamma\left(r_{2}\right)} \int_{t_{1}}^{t_{2}} \int_{x_{1}}^{x_{2}}\left|\log \frac{t_{2}}{s}\right|^{r_{1}-1}\left|\log \frac{x_{2}}{y}\right|^{r_{2}-1} \\
& \cdot\left|g_{i}\left(t_{1}, x_{1}, s, y, u_{1}(s, y), \ldots, u_{n}(s, y)\right)\right| d y d s \leq \frac{1}{\Gamma\left(r_{1}\right) \Gamma\left(r_{2}\right)} \int_{1}^{t_{2}} \int_{1}^{x_{2}}\left|\log \frac{t_{2}}{s}\right|^{r_{1}-1}\left|\log \frac{x_{2}}{y}\right|^{r_{2}-1} \times \varphi_{i}(s, y)
\end{aligned}
$$




$$
\begin{aligned}
& \cdot\left(\left|t_{1}-t_{2}\right|+\left|x_{1}-x_{2}\right|\right) \psi_{i}\left(e_{p}\right) d y d s+\frac{1}{\Gamma\left(r_{1}\right) \Gamma\left(r_{2}\right)} \int_{1}^{t_{1}} \int_{1}^{x_{1}} \mid\left(\log \frac{t_{2}}{s}\right)^{r_{1}-1}\left(\log \frac{x_{2}}{y}\right)^{r_{2}-1} \\
& -\left.\left(\log \frac{t_{1}}{s}\right)^{r_{1}-1}\left(\log \frac{x_{1}}{y}\right)^{r_{2}-1}\left|\left(\sum_{j=0}^{n} Q_{i j}\left(t_{1}, x_{1}, s, y\right)\right) d y d s+\frac{1}{\Gamma\left(r_{1}\right) \Gamma\left(r_{2}\right)} \int_{t_{2}}^{t_{2}} \int_{1}^{x_{2}}\right| \log \frac{t_{2}}{s}\right|^{r_{1}-1}\left|\log \frac{x_{2}}{y}\right|^{r_{2}-1} \\
& \cdot\left(\sum_{j=0}^{n} Q_{i j}\left(t_{1}, x_{1}, s, y\right)\right) d y d s+\frac{1}{\Gamma\left(r_{1}\right) \Gamma\left(r_{2}\right)} \int_{1}^{t_{1}} \int_{x_{1}}^{x_{2}}\left|\log \frac{t_{2}}{s}\right|^{r_{1}-1}\left|\log \frac{x_{2}}{y}\right|^{r_{2}-1}\left(\sum_{j=0}^{n} Q_{i j}\left(t_{1}, x_{1}, s, y\right)\right) d y d s \\
& +\frac{1}{\Gamma\left(r_{1}\right) \Gamma\left(r_{2}\right)} \int_{t_{1}}^{t_{2}} \int_{x_{1}}^{x_{2}}\left|\log \frac{t_{2}}{s}\right|^{r_{1}-1}\left|\log \frac{x_{2}}{y}\right|^{r_{2}-1}\left(\sum_{j=0}^{n} Q_{i j}\left(t_{1}, x_{1}, s, y\right)\right) d y d s .
\end{aligned}
$$

From the continuity of functions $Q_{i j}, \varphi_{i}$ and as $t_{1} \longrightarrow t_{2}$ and $x_{1} \longrightarrow x_{2}$, the right-hand side of the above inequality tends to zero. As a consequence of Claims 1-3 and from the ArzeláAscoli theorem, we can conclude that $A$ is continuous and compact.

Step 2 ( $B$ is a contraction). Consider $\left(v_{1}, \ldots, v_{n}\right),\left(w_{1}, \ldots\right.$, $\left.w_{n}\right) \in \mathscr{C}$. Then, by $\left(H_{1}\right)$, for any $p \in \mathbb{N} \backslash\{0,1\}$ and each $(t, x) \in[1, p] \times[1, b]$ and $i=1, \ldots, n$, we have

$$
\begin{aligned}
& \left|\left(B_{i} v_{i}\right)(t, x)-\left(B_{i} w_{i}\right)(t, x)\right| \\
& \leq\left. l_{i}(t, x)\right|^{H} I_{\sigma}^{r}\left(v_{i}-w_{i}\right)(t, x) \mid \\
& \quad+k_{i}(t, x)\left|\left(v_{i}-w_{i}\right)(t, x)\right| \\
& \leq\left(k_{i p}+\frac{l_{i p}(\log p)^{r_{1}}(\log b)^{r_{2}}}{\Gamma\left(1+r_{1}\right) \Gamma\left(1+r_{2}\right)}\right)\left\|v_{i}-w_{i}\right\|_{p} \\
& \leq\left(k_{p}^{*}+\frac{l_{p}^{*}(\log p)^{r_{1}}(\log b)^{r_{2}}}{\Gamma\left(1+r_{1}\right) \Gamma\left(1+r_{2}\right)}\right)\left\|v_{i}-w_{i}\right\|_{p} .
\end{aligned}
$$

Thus,

$$
\begin{aligned}
& \sum_{i=1}^{n}\left|\left(B_{i} v_{i}\right)(t, x)-\left(B_{i} w_{i}\right)(t, x)\right| \\
& \quad \leq\left(k_{p}^{*}+\frac{l_{p}^{*}(\log p)^{r_{1}}(\log b)^{r_{2}}}{\Gamma\left(1+r_{1}\right) \Gamma\left(1+r_{2}\right)}\right) \sum_{i=1}^{n}\left\|v_{i}-w_{i}\right\|_{p} .
\end{aligned}
$$

Hence,

$$
\begin{aligned}
& \|\left(B\left(v_{1}, \ldots, v_{n}\right)-B\left(w_{1}, \ldots, w_{n}\right) \|_{p}\right. \\
& \quad \leq L_{p}\left\|\left(v_{1}, \ldots, v_{n}\right)-\left(w_{1}, \ldots, w_{n}\right)\right\|_{p} .
\end{aligned}
$$

Inequality (21) implies that $B$ is a contraction operator.
Step 3 (the set $\mathscr{E}:=\left\{u=\left(u_{1}, \ldots, u_{n}\right) \in \mathscr{C}: u=\lambda A(u)+\right.$ $\lambda B(u / \lambda), \lambda \in(0,1)\}$ is bounded). Let $\left(u_{1}, \ldots, u_{n}\right) \in \mathscr{C}$, such that $\left(u_{1}, \ldots, u_{n}\right)=\lambda A\left(u_{1}, \ldots, u_{n}\right)+\lambda B\left(u_{1} / \lambda, \ldots, u_{n} / \lambda\right)$, for some $\lambda \in(0,1)$. Then, for any $p \in \mathbb{N} \backslash\{0,1\}$ and each $(t, x) \in$ $[1, p] \times[1, b]$ and $i=1, \ldots, n$, we have

$$
\begin{aligned}
& \left|u_{i}(t, x)\right| \leq \lambda\left|A_{i}\left(u_{i}\right)\right|+\lambda\left|B_{i}\left(\frac{u_{i}}{\lambda}\right)\right| \leq\left|\mu_{i}(t, x)\right| \\
& +\left|f_{i}(t, x, 0,0)\right|+k_{i}(t, x)+l_{i}(t, x)+\frac{1}{\Gamma\left(r_{1}\right) \Gamma\left(r_{2}\right)} \\
& \quad \cdot \int_{1}^{t} \int_{1}^{x}\left|\log \frac{t}{s}\right|^{r_{1}-1}\left|\log \frac{x}{y}\right|^{r_{2}-1} \\
& \quad \times\left(\sum_{j=0}^{n} Q_{i j}(t, x, s, y)\right) \frac{d y d s}{s y} \leq \mu_{i p}+f_{i p} \\
& +k_{i p}+l_{i p}+\sum_{j=0}^{n} Q_{i j p},
\end{aligned}
$$

where

$$
\begin{aligned}
& \mu_{i p}=\sup _{(t, x) \in[1, p] \times[1, b]}\left|\mu_{i}(t, x)\right|, \\
& f_{i p}=\sup _{(t, x) \in[1, p] \times[1, b]}|f(t, x, 0,0)| ;
\end{aligned}
$$

$p \in \mathbb{N} \backslash\{0,1\}$

Thus,

$$
\left\|\left(u_{1}, \ldots, u_{n}\right)\right\|_{p} \leq \mu_{p}^{*}+f_{p}^{*}+k_{p}^{*}+l_{p}^{*}+Q_{p}^{*}=: \ell_{p}^{*} .
$$

Hence, the set $\mathscr{E}$ is bounded. As a consequence of Steps 1-3 and from Theorem 5, we deduce that $N$ has a fixed point $\left(u_{1}, \ldots, u_{n}\right)$ which is a solution of system (2). 


\section{An Example}

Consider the following coupled system of Hadamard fractional integral equations of the form

$$
\begin{aligned}
& u(t, x)=\frac{x e^{3-2 t}}{1+t+x^{2}} \\
& +\frac{x e^{-t-2}}{c_{p}\left(1+e^{-2 p}\left|\left({ }^{H} I_{\sigma}^{r} u\right)(t, x)\right|+e^{-p}|u(t, x)|\right)} \\
& +\int_{1}^{t} \int_{1}^{x}\left(\log \frac{t}{s}\right)^{r_{1}-1}\left(\log \frac{x}{y}\right)^{r_{2}-1} \\
& . \frac{g_{1}(t, x, s, y, u(s, y), v(s, y))}{\Gamma\left(r_{1}\right) \Gamma\left(r_{2}\right)} d y d s \\
& +t, x)=\frac{1}{1+e^{t+x}} \quad x e^{-t-2} \\
& +\int_{1}^{t} \int_{1}^{x}\left(\log \frac{t}{s}\right)^{r_{1}-1}\left(\log \frac{x}{y}\right)^{r_{2}-1} \\
& \quad . \frac{g_{2}(t, x, s, y, u(s, y), v(s, y))}{\Gamma\left(r_{1}\right) \Gamma\left(r_{2}\right)} d y d s ; \\
& \quad+(t, x) \in[1,+\infty) \times[1, e]
\end{aligned}
$$

where $c_{p}=e^{-p}+e^{-2 p} p^{r_{1}} / \Gamma\left(1+r_{1}\right) \Gamma\left(1+r_{2}\right) ; p \in \mathbb{N} \backslash\{0,1\}$, $r=\left(r_{1}, r_{2}\right) \in(0, \infty) \times(0, \infty)$, and

$$
\begin{aligned}
g_{1}(t, x, s, y, u, v)= & \frac{x s^{-3 / 4} \sin \sqrt{t} \sin s}{\left(1+y^{2}+t^{2}\right)(1+|u|+|v|)} ; \\
& \text { if }(t, x, s, y) \in J^{\prime}, \text { and } u \in \mathbb{R}, \\
g_{2}(t, x, s, y, u, v)= & \frac{s^{-3 / 4} \ln \sqrt{t}}{\left(1+t^{2}\right)(1+|u|+|v|)} ; \\
& \text { if }(t, x, s, y) \in J^{\prime}, \text { and } u \in \mathbb{R},
\end{aligned}
$$

and

$$
J^{\prime}=\{(t, x, s, y): 1 \leq s \leq t \text { and } 1 \leq x \leq y \leq e\} .
$$

Set

$$
\begin{aligned}
\mu_{1}(t, x) & =\frac{x e^{3-2 t}}{1+t+x^{2}}, \\
\mu_{2}(t, x) & =\frac{1}{1+e^{t+x}}, \\
f_{1}(t, x, u, v) & =\frac{x e^{-t-2}}{c_{p}\left(1+e^{-2 p}|u|+e^{-p}|u|\right)}, \\
f_{2}(t, x, u, v) & =\frac{x e^{-t-2}}{c_{p}\left(1+e^{-2 p}|v|+e^{-p}|v|\right)}
\end{aligned}
$$$$
p \in \mathbb{N} \backslash\{0,1\} .
$$

The functions $f_{i} ; i=1,2$ are continuous and satisfy assumption $\left(H_{1}\right)$, with

$$
\begin{aligned}
k_{1}(t, x) & =k_{2}(t, x)=\frac{x e^{-t-2-p}}{c_{p}}, \\
l_{1}(t, x) & =l_{2}(t, x)=\frac{x e^{-t-2-2 p}}{c_{p}}, \\
k_{p} & =\frac{e^{-2-p}}{c_{p}}, \\
l_{p} & =\frac{e^{-2-2 p}}{c_{p}} .
\end{aligned}
$$

Also, the functions $g_{i}, 1=1,2$ are continuous and satisfy assumption $\left(\mathrm{H}_{2}\right)$, with

$$
\begin{array}{r}
Q_{i 0}(t, x, s, y)=Q_{i 1}(t, x, s, y)=\frac{x s^{-3 / 4} \sin \sqrt{t} \sin s}{1+y^{2}+t^{2}} \\
(t, x, s, y) \in J^{\prime} ; i=1,2 .
\end{array}
$$

Then,

$$
\begin{aligned}
& \left.\left|\int_{1}^{t}\right| \log \frac{t}{s}\right|^{r_{1}-1} \frac{Q_{01}(t, x, s, y)}{s} d s \mid \\
& \quad \leq \int_{1}^{t}\left|\log \frac{t}{s}\right|^{r_{1}-1} x s^{-1}|\sin \sqrt{t} \sin s| d s \\
& \quad \leq\left. x\left|\sin \sqrt{t} \int_{1}^{t}\right| \log \frac{t}{s}\right|^{r_{1}-1} s^{-1} d s \leq e\left|\frac{(\log t)^{r_{1}}}{\sqrt{t}}\right| \\
& \quad \longrightarrow 0 \text { as } t \longrightarrow \infty .
\end{aligned}
$$

Finally, we shall show that condition (21) holds with $b=e$. Indeed, for each $p \in \mathbb{N} \backslash\{0,1\}$, we get

$$
\begin{aligned}
L_{p} & =k_{p}^{*}+\frac{l_{p}^{*}(\log p)^{r_{1}}(\log b)^{r_{2}}}{\Gamma\left(1+r_{1}\right) \Gamma\left(1+r_{2}\right)} \\
& =\frac{1}{c_{p}}\left(e^{-2-p}+\frac{e^{-2-2 p} p^{r_{1}}}{\Gamma\left(1+r_{1}\right) \Gamma\left(1+r_{2}\right)}\right)=e^{-2}<1 .
\end{aligned}
$$

Hence by Theorem 7, system (39) has a solution defined on $[1,+\infty) \times[1, e]$.

\section{Data Availability}

No data were used to support this study.

\section{Conflicts of Interest}

The authors declare that there are no conflicts of interest regarding the publication of this paper.

\section{Acknowledgments}

The work was supported by the National Natural Science Foundation of China (No. 11671339). 


\section{References}

[1] S. Abbas, M. Benchohra, and G. M. N’Guérékata, Topics in Fractional Differential Equations, Springer, New York, NY, USA, 2012.

[2] S. Abbas, M. Benchohra, and G. M. N'Guérékata, Advanced Fractional Differential and Integral Equations, Nova Science Publishers, New York, NY, USA, 2015.

[3] D. Baleanu, K. Diethelm, E. Scalas, and J. J. Trujillo, Fractional Calculus Models and Numerical Methods, vol. 3 of Series on Complexity, Nonlinearity and Chaos, World Scientific, 2012.

[4] A. A. Kilbas, H. M. Srivastava, and J. J. Trujillo, Theory and Applications of Fractional Differential Equations, New York, NY, USA, Elsevier, 2006.

[5] V. Lakshmikantham, S. Leela, and J. Vasundhara, Theory of Fractional Dynamic Systems, Cambridge Academic Publishers, Cambridge, 2009.

[6] F. H. Miller and B. Ross, An Introduction to the Fractional Calculus and Differential Equations, John Wiley and Sons Inc., New York, NY, USA, 1993.

[7] S. G. Samko, A. A. Kilbas, and O. I. Marichev, Fractional Integrals and Derivatives, Theory and Applications, Gordon and Breach, Yverdon, Switzerland, 1993.

[8] B. Ahmad, A. Alsaedi, and M. Kirane, "Nonexistence results for the Cauchy problem of time fractional nonlinear systems of thermo-elasticity," Mathematical Methods in the Applied Sciences, vol. 40, no. 12, pp. 4272-4279, 2017.

[9] J. Wang, M. Feckan, and Y. Zhou, "A survey on impulsive fractional differential equations," Fractional Calculus and Applied Analysis, vol. 19, no. 4, pp. 806-831, 2016.

[10] J. Wang, M. Feckan, and Y. Zhou, "Center stable manifold for planar fractional damped equations," Applied Mathematics and Computation, vol. 296, pp. 257-269, 2017.

[11] Y. Zhou, "Attractivity for fractional differential equations in Banach space," Applied Mathematics Letters, vol. 75, pp. 1-6, 2018.

[12] Y. Zhou, "Attractivity for fractional evolution equations with almost sectorial operators," Fractional Calculus and Applied Analysis, vol. 21, no. 3, pp. 786-800, 2018.

[13] Y. Zhou, B. Ahmad, and A. Alsaedi, "Existence of nonoscillatory solutions for fractional neutral differential equations," Applied Mathematics Letters, vol. 72, pp. 70-74, 2017.

[14] Y. Zhou, L. Shangerganesh, J. Manimaran, and A. Debbouche, "A class of time-fractional reaction-diffusion equation with nonlocal boundary condition," Mathematical Methods in the Applied Sciences, vol. 41, no. 8, pp. 2987-2999, 2018.

[15] Y. Zhou, L. Peng, B. Ahmad, and A. Alsaedi, “Topological properties of solution sets of fractional stochastic evolution inclusions," Advances in Difference Equations, p. 90, 2017.

[16] Y. Zhou, V. Vijayakumar, and R. Murugesu, "Controllability for fractional evolution inclusions without compactness," Evolution Equations and Control Theory, vol. 4, no. 4, pp. 507-524, 2015.

[17] Y. Zhou and L. Zhang, "Existence and multiplicity results of homoclinic solutions for fractional Hamiltonian systems," Computers \& Mathematics with Applications, vol. 73, no. 6, pp. 1325-1345, 2017.

[18] Y. Zhou, L. Zhang, and X. H. Shen, "Existence of mild solutions for fractional evolution equations," Journal of Integral Equations and Applications, vol. 25, no. 4, pp. 557-586, 2013.

[19] P. L. Butzer, A. A. Kilbas, and J. J. Trujillo, "Fractional calculus in the Mellin setting and Hadamard-type fractional integrals,"
Journal of Mathematical Analysis and Applications, vol. 269, no. 1, pp. 1-27, 2002.

[20] P. L. Butzer, A. A. Kilbas, and J. J. Trujillo, "Mellin transform analysis and integration by parts for Hadamard-type fractional integrals," Journal of Mathematical Analysis and Applications, vol. 270, no. 1, pp. 1-15, 2002.

[21] S. Pooseh, R. Almeida, and D. F. Torres, "Expansion formulas in terms of integer-order derivatives for the Hadamard fractional integral and derivative," Numerical Functional Analysis and Optimization, vol. 33, no. 3, pp. 301-319, 2012.

[22] S. Abbas, W. Albarakati, and M. Benchohra, "Successive approximations for functional evolution equations and inclusions," Journal of Nonlinear Functional Analysis, vol. 2017, no. 39, pp. $1-13,2017$.

[23] S. Abbas and M. Benchohra, "Fractional order RiemannLiouville integral equations with multiple time delays," Applied Mathematics E-Notes, vol. 12, pp. 79-87, 2012.

[24] S. Abbas, M. Benchohra, J.-E. Lazreg, and Y. Zhou, "A survey on Hadamard and HILfer fractional differential equations: analysis and stability," Chaos, Solitons \& Fractals, vol. 102, pp. 47-71, 2017.

[25] J. Banaś and T. Zajaç, "Solvability of a functional integral equation of fractional order in the class of functions having limits at infinity," Nonlinear Analysis: Theory, Methods \& Applications, vol. 71, no. 11, pp. 5491-5500, 2009.

[26] J. Banaś and T. Zając, "A new approach to the theory of functional integral equations of fractional order," Journal of Mathematical Analysis and Applications, vol. 375, no. 2, pp. 375387, 2011.

[27] B. G. Pachpatte, "On Volterra-Fredholm integral equation in two variables," Demonstratio Mathematica, vol. 40, no. 4, pp. 839-852, 2007.

[28] B. G. Pachpatte, "On Fredholm type integral equation in two variables," Differential Equations \& Applications, vol. 1, no. 1, pp. 27-39, 2009.

[29] M. Frigon and A. Granas, “Théorèmes d'existence pour des inclusions différentielles sans convexité," Comptes rendus de l'Académie des Sciences, vol. 310, no. 12, pp. 819-822, 1990.

[30] C. Avramescu, "Some remarks on a fixed point theorem of Krasnoselskii," Electronic Journal of Qualitative Theory of Differential Equations, No. 5, 15 pages, 2003. 


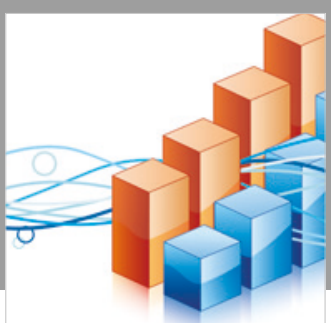

Advances in

Operations Research

\section{-n-m}
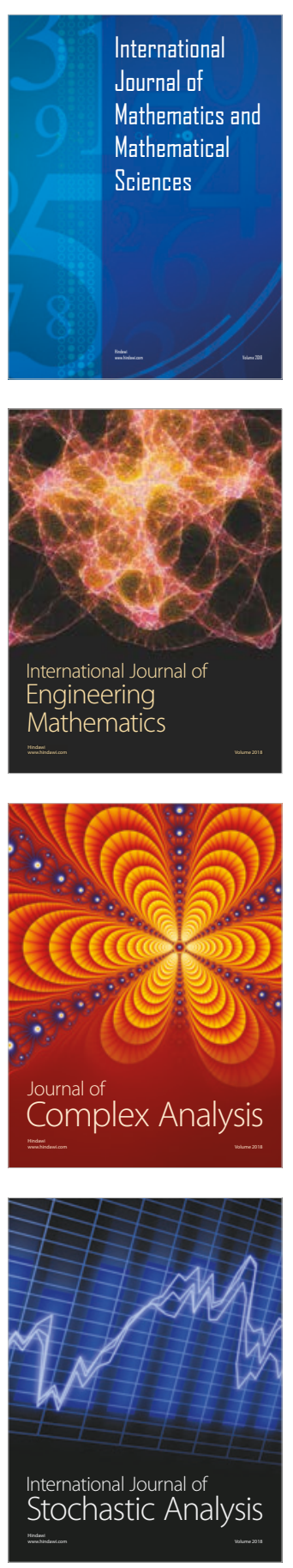
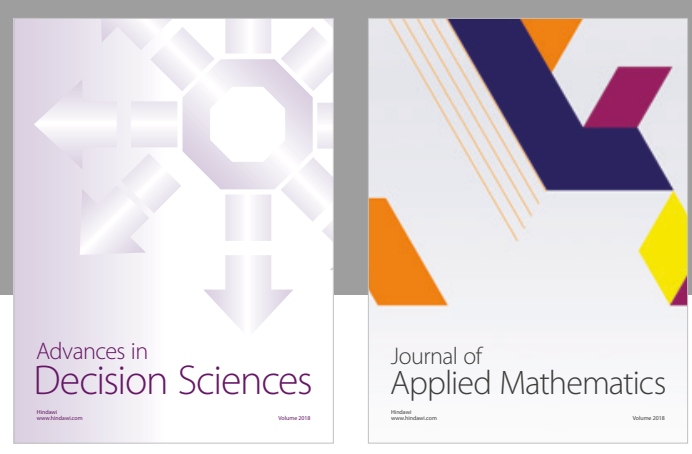

Journal of

Applied Mathematics
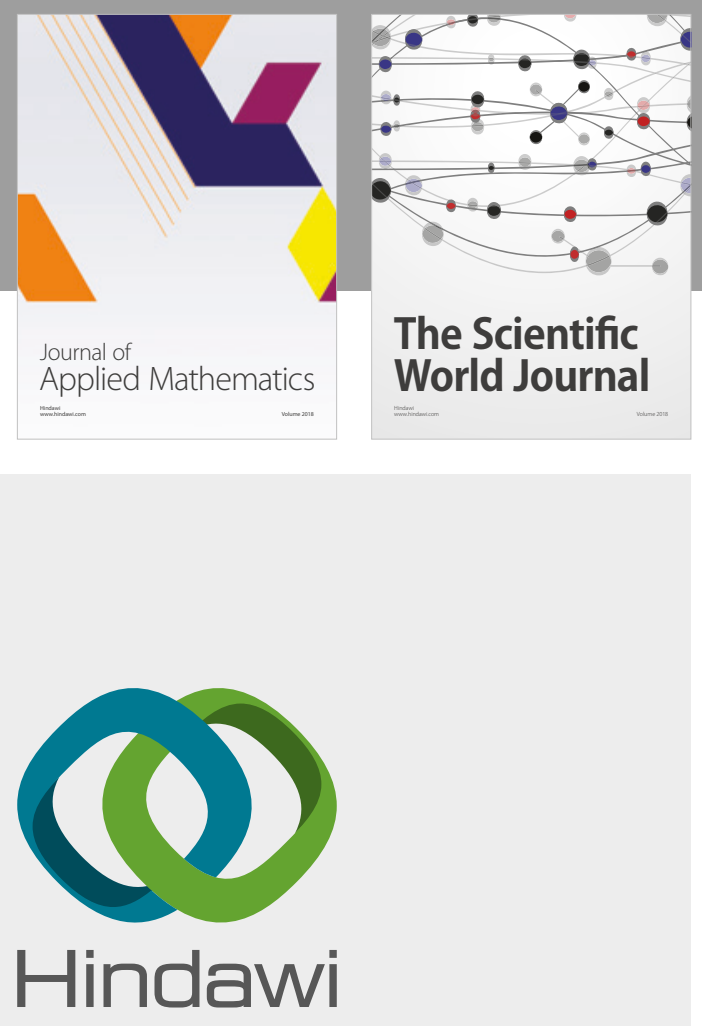

Submit your manuscripts at

www.hindawi.com

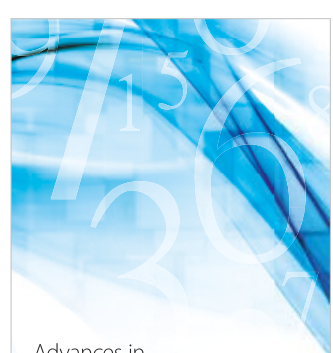

Advances in
Numerical Analysis
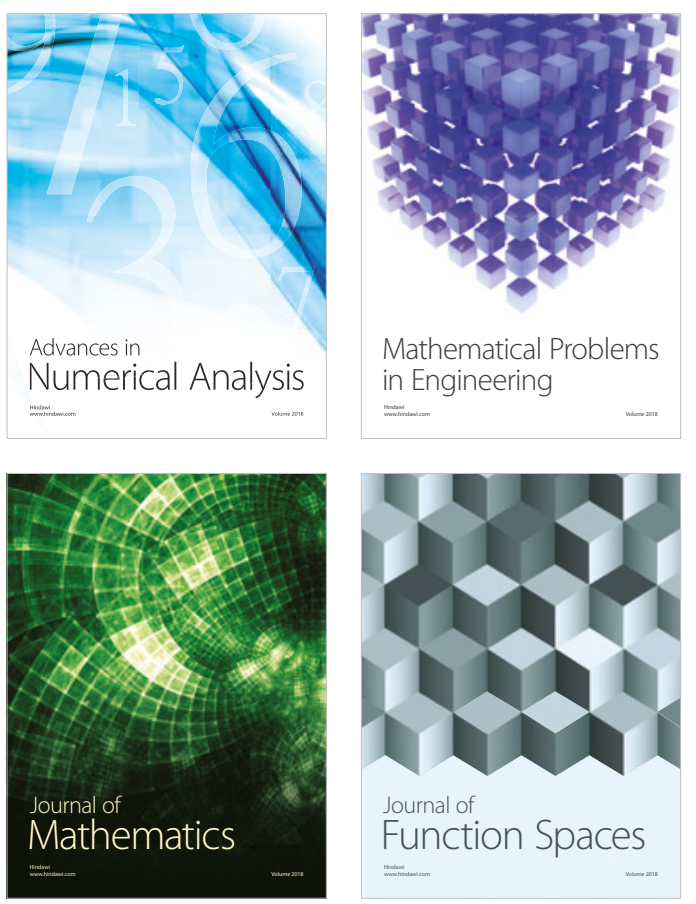

Mathematical Problems in Engineering

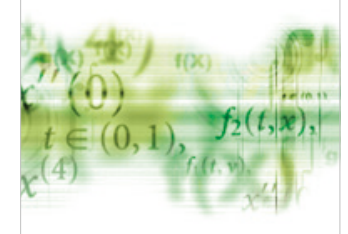

International Journal of

Differential Equations

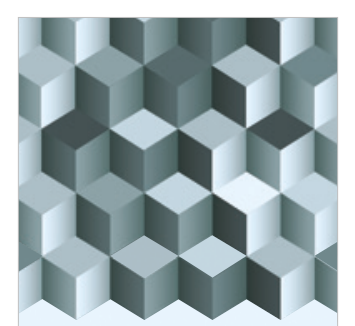

Journal of

Function Spaces

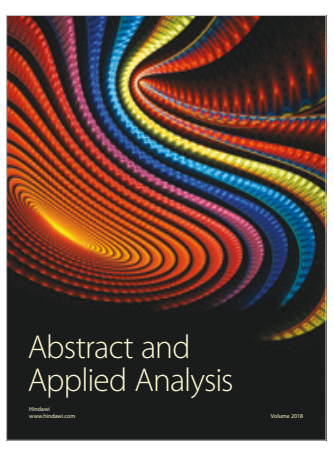

The Scientific

World Journal

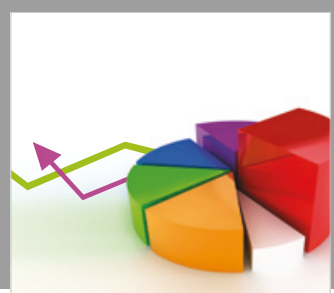

Journal of

Probability and Statistics
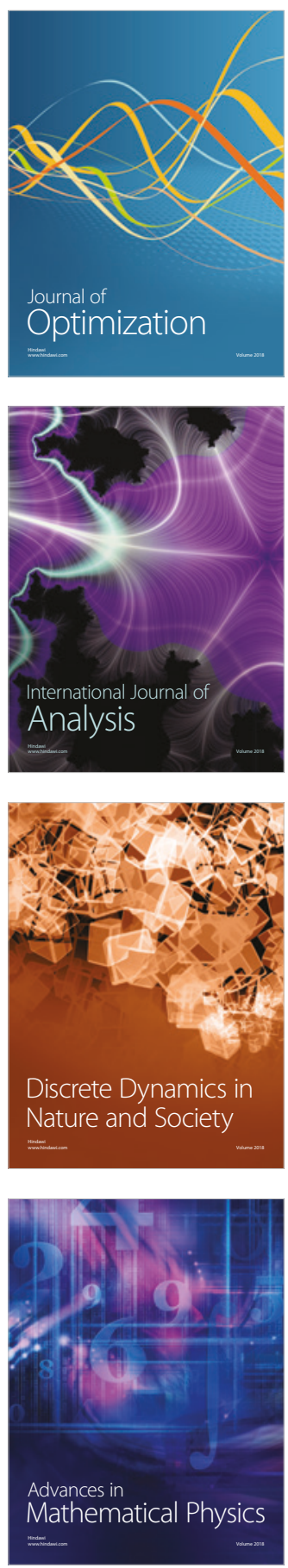